\title{
The study of genetic diversity of flat leeches
}

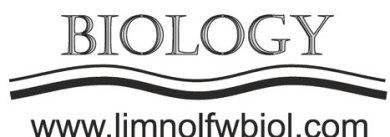

\author{
Bolbat N.B. ${ }^{1,2 *}$, Kaygorodova I.A. ${ }^{1}$ \\ ${ }^{1}$ Limnological Institute, Siberian Branch of the Russian Academy of Sciences, Ulan-Batorskaya Str., 3, Irkutsk, 664033, Russia \\ ${ }^{2}$ Irkutsk State University, Sukhe-Bator Str., 5, Irkutsk, 664011, Russia
}

\begin{abstract}
The conservation of species is one of the most important tasks of biology, which involves a comprehensive study and determination of the taxonomic hierarchy of various representatives of the fauna. Species composition of flat leeches requires thorough revision, as evidenced by ambiguous morphological descriptions due to anatomic features of glossiphoniids, which complicates the taxonomic identification of leeches of this group. The combination of methods of morphological identification and molecular genetic analysis allowed us to prove the belonging of A.hyalina as a third form of the species A.heteroclita along with A.heteroclita f.papillosa and A.heteroclita f.striata. Intraspecies genetic variability was $0.2 \%$, which confirmed the genetic homogenity of the leeches of this species. The results obtained during the study demonstrate the effectiveness of the genetic diversity analysis within the comprehensive approach to the study of the species composition of flat leeches.
\end{abstract}

Keywords: Glossiphoniidae, DNA barcoding, genetic diversity

\section{Introduction}

Flat leeches (Glossiphoniidae) have a wide geographic distribution and play an important ecological and parasitological role in the ecosystem, which is why they are of great interest from the global scientific community. At the same time, species identification of flat leeches is a subject for discussion, because the diagnosis of morphological characters often does not take into account the level of their intraspecific variability. This results in inaccurate ideas about the characteristics of taxonomic groups. Leeches of the genus Alboglossiphonia (Lukin, 1976) are one of such examples. The genus Alboglossiphonia currently consists of three species: A.hyalina, A.heteroclita and A.weberi. At the same time, the taxonomic position of $A$. heteroclita and A. hyalina is disputable due to the ambiguity of the distinctive features for establishing an independent species status. Previously, these species were described as the species $A$. heteroclita with the striata and papillosa forms, where A. hyalina was included as the third form (Lukin, 1976). Although all three forms have differences in pigment patterns on the dorsal surface of the body, they do not have anatomic differences and occupy one ecological niche.

Currently, to resolve this situation, it is possible to apply a comprehensive approach that includes morphological diagnosis and molecular genetic method for species identification - DNA barcoding (Hebert and Gregory, 2005).

\section{Materials and methods}

Biological material was collected in a timespan from 2014 to 2019 on the territory of East Siberia. Morphological analysis was carried out according to modern identification keys (Lukin, 1976; Nesemann and Neubert, 1999). Molecular genetic analysis was carried out through DNA extraction according to the standard method of phenol-free extraction followed by amplification of the COI fragment using the universal primers. Sequencing was carried out at Syntol company (Moscow, Russia). The phylogeny was reconstructed using the Tamura-Nei model with the maximum likelihood (ML) method implemented in MEGA 7.0 (Kumar et al., 2016).

\section{Results and discussion}

During the study, we obtained the sequences of the Folmer COI region with a length of $709 \mathrm{bp}$ for 15 leeches from different water bodies. The 16 closest homologous sequences from GenBank were included in the analysis as a reference group. The sequences of the genus Glossiphonia were used as an outgroup. Inside the cluster of nucleotide sequences of A.heteroclita f.papillosa, A.heteroclita f.striata and A.hyalina, molecular genetic analysis indicated a low level of genetic variability that did not exceed $0.2 \%$ (Fig.). Based on the obtained data, we can assume that

*Corresponding author.

E-mail address: nadya.mandzyak@mail.ru (N.B. Bolbat) 


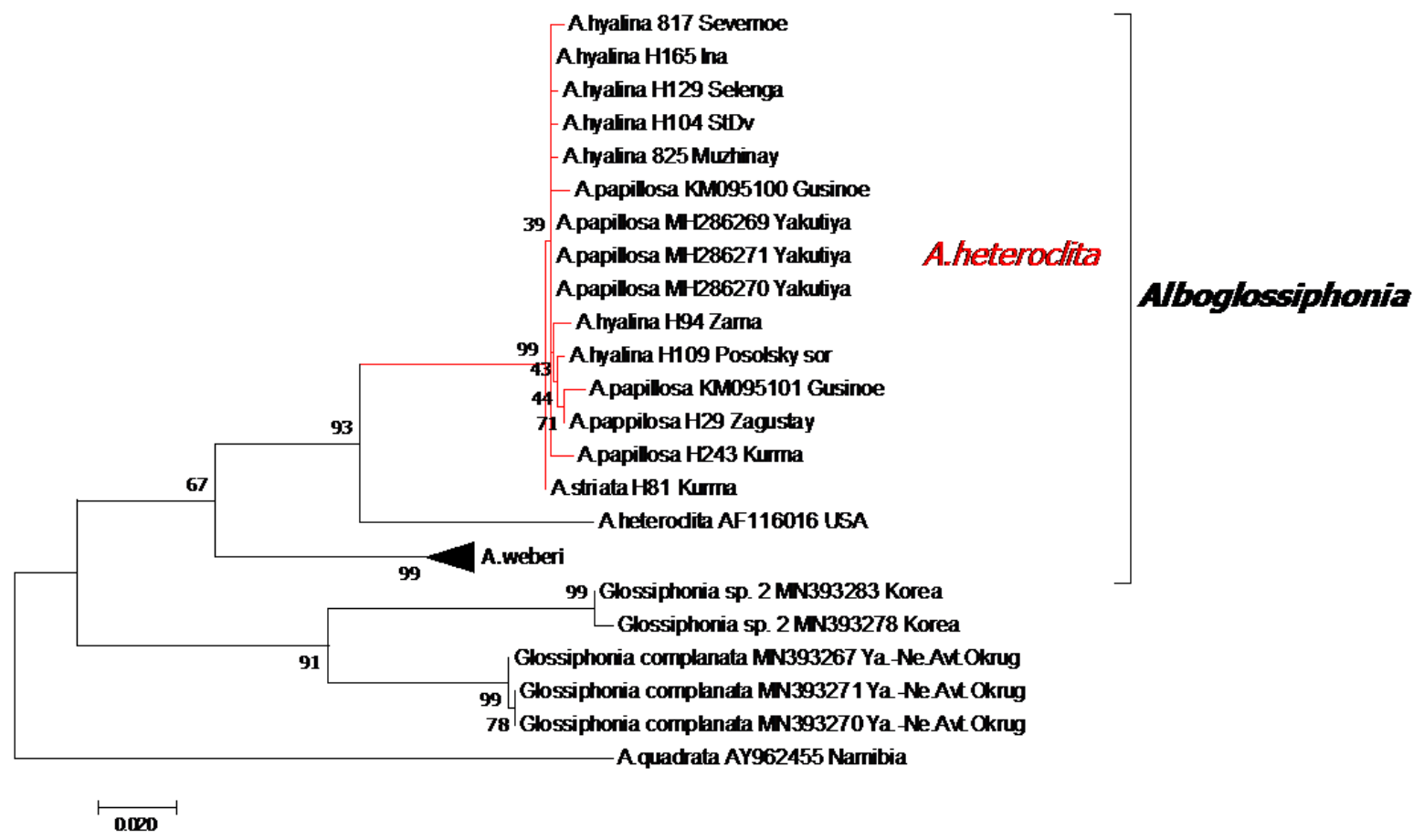

Fig. ML-tree $(\operatorname{lnL}=-2477,56)$ based on 31 COI nucleotide sequences.

leeches A.heteroclita f.papillosa, A.heteroclita f.striata, and A.hyalina are three forms of the same species A.heteroclita as previously stated (Lukin, 1976). At the same time, the genetic distance between the members of the Siberian species and the sample from the USA taken from the genetic database is $7.1 \%$, which exceeds the threshold value and confirms the independent species status of these leeches.

The results obtained confirm the effectiveness of the comprehensive approach to species identification of flat leeches.

\section{Acknowledgements}

This work was supported by the by RFBR grant №. 17-29-05097.

\section{References}

Hebert P., Gregory T.R. 2005. The promise of DNA barcoding for taxonomy. Systematic Biology 54: 852-859. DOI: 10.1080/10635150500354886

Kumar S., Stecher G., Tamura K. 2016. MEGA7: Molecular Evolutionary Genetics Analysis version 7.0 for bigger datasets. Molecular Biology and Evolution 33: 18701874. DOI: $10.1093 / \mathrm{molbev} / \mathrm{msw} 054$

Lukin E.I. 1976. Fauna SSSR. Piyavki: Tom I. Piyavki presnovodnykh i solonovatykh vodoyomov [The USSR fauna. Leeches: Vol. I. Freshwater and brackish water leeches]. Leningrad: Nauka. (in Russian)

Nesemann H., Neubert E. 1999. Clitellata, Branchiobdellada, Acanthobdellada, Hirudinea. In: Schwoebel J., Zwig P. (Eds.), Süßwasserfauna von Mitteleuropa: Vol. 6(2) [Freshwater fauna of Central Europe: Vol. 6(2)]. Heidelberg: Spectrum Akademischer Verlag. 